\title{
THE EQUIVARIANT CONNER-FLOYD ISOMORPHISM
}

\author{
STEVEN R. COSTENOBLE
}

\begin{abstract}
This paper proves two equivariant generalizations of the ConnerFloyd isomorphism relating unitary cobordism and $K$-theory. It extends a previous result of Okonek for abelian groups to all compact Lie groups. We also show that the result for finite groups is true using either the geometric or homotopical versions of cobordism.
\end{abstract}

1. Introduction. In [6] Conner and Floyd established a relation between cobordism and $K$-theory. They proved that

$$
\widetilde{M U}^{*}(X) \otimes_{M U^{*}} K^{*} \cong \tilde{K}^{*}(X),
$$

where $M U$ is unitary cobordism and $K$ is complex $K$-theory. A generalization of this result to the equivariant context was proved by Okonek [15]. This paper improves upon that generalization in two ways. First, it expands the class of groups considered, from abelian groups to all compact Lie groups. Second, it shows that both of the usual generalizations of cobordism to the equivariant context can be used.

The $K$-theory that we use is $K_{G}^{*}(-)$, the equivariant $K$-theory of Segal [17], which is the obvious generalization of $K$-theory to the equivariant world. Atiyah [2] proved that this theory has Bott periodicity; it is periodic with respect to $\mathrm{Spin}^{c}$-representations of the group. There are two common ways of generalizing cobordism; we will define these carefully in $\S 2$. One gives geometric cobordism, which we denote by $U_{G}^{*}(-)$, and the other homotopical cobordism, $M U_{G}^{*}(-)$. Both of these are multiplicative cohomology theories. There are multiplicative maps $\mu: \mathcal{U}_{G}^{*}(-) \rightarrow K_{G}^{*}(-)$ and $\mu: M U_{G}^{*}(-) \rightarrow K_{G}^{*}(-)$. Writing $\mathcal{U}_{G}^{*}$ for the ring $\mathcal{U}_{G}^{*}(*)$, and similarly for the other theories, the two main results we will show are

THEOREM A. $\mu: \tilde{U}_{G}^{*}(X) \otimes u_{G}^{*} K_{G}^{*} \rightarrow \tilde{K}_{G}^{*}(X)$ is an isomorphism when $G$ is a finite group.

THEOREM B. $\mu: \widetilde{M U}_{G}^{*}(X) \otimes_{M U_{G}^{*}} K_{G}^{*} \rightarrow \tilde{K}_{G}^{*}(X)$ is an isomorphism for every compact Lie group $G$.

It should be pointed out that these results use integer grading and not $R O(G)$ grading. We will use integer grading throughout this paper, except on those occasions when it is necessary or particularly convenient to use $R O(G)$; we will make it clear at these points that we are using the larger grading.

Theorem B has already been shown for $G$ abelian in [15], and we will use that result as a starting point. Theorem A follows from Theorem B, as shown in $\S 3$, by

Received by the editors March 4, 1986 and, in revised form, September 8, 1986.

1980 Mathematics Subject Classification (1985 Revision). Primary 57R85. 
an argument due to Ib Madsen. He and Mel Rothenberg have used Theorem A in work on the homotopy groups of equivariant automorphism groups of spheres.

The proof of Theorem B is similar in outline to Atiyah and Segal's proof of the completion theorem for $K$-theory [3]. By embedding $G$ in $U(n)$, we can reduce to the case where $G$ is $U(n)$. By a suitable transfer, we can relate $U(n)$ to its maximal torus $T^{n}$, for which the result is already known. To complete the proof we must delve into the structure of the equivariant cobordism rings. The proof of Theorem B occupies $\S \S 4$ and 5 .

These results were the main ones of my Ph.D. thesis. I would like to thank my advisor, J. Peter May, for his helpful guidance. I would also like to thank Ib Madsen for his permission to reproduce the argument of $\S 3$ here.

2. Definitions. Before we begin serious work, we establish terminology. Throughout this paper we will be concerned with group actions; the group which is acting will always be assumed to be compact Lie, and most of the actions will be on the left. Actions on smooth manifolds will be assumed to be smooth. When we refer to a subgroup of a compact Lie group, we always mean a closed subgroup. A representation of a compact Lie group $G$ is a finite-dimensional real vector space on which $G$ acts linearly; we may assume that it has a $G$-invariant inner product. If $V$ is a representation of $G$, then

$$
D(V)=\{v \in V \mid\|v\| \leq 1\} \quad \text { and } \quad S(V)=\{v \in V \mid\|v\|=1\}
$$

are the unit disc and sphere of $V . S^{V}$ denotes the one-point compactification of $V$, with compactification point as basepoint. $\Sigma^{V} X=X \wedge S^{V}$ for any based $G$-space $X$. $|V|$ will always mean the real dimension of $V$. If $W \subset V$ is a subrepresentation, then $V-W$ denotes the orthogonal complement to $W$ in $V$. In particular, $V_{H}=V-V^{H}$, the $H$-nontrivial part of $V . R O(G)$ will denote the real representation $\operatorname{ring}$ of $G$, and $R(G)$ the complex representation ring. $\mathrm{Z} \subset R O(G)$ is the subring of trivial representations, and $\mathbf{R}^{n}$ is the trivial representation of dimension $n$.

If $X$ is any $G$-space, $X^{+}$will denote $X$ with a $G$-fixed disjoint basepoint attached. Basepoints will in general be referred to as "*," as will any one-point space. If $H$ is a subgroup of $G$ and $X$ is a based $H$-space, then the extension of $X$ to $G$ is the $G$ space $G^{+} \wedge_{H} X=\left(G^{+} \wedge X\right) / H$ where $H$ acts on the right by $(g, x) h=\left(g h, h^{-1} x\right)$. $G$ acts on the left via its action on itself by multiplication. If $X$ and $Y$ are based $G$-spaces, then $[X, Y]_{G}$ will denote the set of based $G$-homotopy classes of based $G$-maps from $X$ to $Y$.

We will have occasion to take limits over "all representations of $G$." To make sense of this, we employ a complete $G$-universe, which is a countably infinitedimensional $G$-inner product space which contains every finite-dimensional representation of $G$ infinitely many times. A limit over all of the representations of $G$ is then interpreted as a limit over the finite-dimensional subspaces of such a universe, directed by inclusion.

2.1 Geometric cobordism. We now define our cobordism theories. Unfortunately, there are several different generalizations of unitary cobordism to the equivariant world. The most obvious, and the first we will define, is the geometric version. 
We start by defining what a unitary $G$-manifold is, and here again we have some choices. Our preferred definition is this:

DEFINITION. A unitary structure on a smooth closed $G$-manifold $M$ is an embedding of $M$ in a $G$-representation $V \oplus \mathbf{R}^{n}$, where $V$ is a complex $G$-representation, together with a complex structure on the normal bundle of this embedding. An equivalence relation is defined on unitary structures in the familiar way, via additions of complex representations to $V \oplus \mathbf{R}^{n}$ (and the normal bundle), and isotopies of embeddings. A unitary $G$-manifold is a $G$-manifold together with an equivalence class of unitary structures on it.

We will refer to a representation of the form $V \oplus \mathbf{R}^{n}$, where $V$ is a complex representation, as a complex $\oplus$ trivial representation. An equivalent way of defining a unitary manifold would be to require that the stable tangent bundle of $M$ be given a complex structure, if we understand stabilization to mean addition of complex $\oplus$ trivial representations. Many writers use a more restrictive definition, which allows only trivial representations to be added to the tangent bundle. For many of our arguments, both definitions will work and lead to the same result. Our preferred definition has the advantage that it leads to a homology theory which can be reasonably easily represented by a spectrum, and hence leads to a corresponding cohomology theory, as explained below.

One more comment on the definition is in order. The representation $V \oplus \mathbf{R}^{n}$ which appears could have been specified to have $n$ equal to 0 or 1 ; other copies of $\mathbf{R}$ can be paired into copies of $\mathbf{C}$ and absorbed into $V$. The choice of one alternative over the other is purely an aesthetic decision. The trivial summands are there at all, of course, to allow odd-dimensional manifolds to be considered to be unitary.

Given a definition of unitary $G$-manifolds, we can define unitary cobordism of such in the usual way. We can also consider manifolds and cobordisms over a $G$-space $X$, meaning that there are $G$-maps from the manifolds and cobordisms into $X$. The set of unitary cobordism classes of $n$-dimensional unitary $G$-manifolds over $X$ is a group which we will call $\mathcal{U}_{n}^{G}(X)$. With an appropriate definition for the relative case, the collection of functors $U_{*}^{G}(-)$ defines a $G$-homology theory, as is well known [5]. We call this geometric unitary bordism. As has also become known, this theory can be represented by a $G$-spectrum, though not quite the one we might expect at first (see $[\mathbf{1 8}, \mathbf{1 9}$, or 7]). Having such a spectrum, we can define an associated $G$-cohomology theory, which we denote by $\mathcal{U}_{G}^{*}(-)$ and which we call geometric unitary cobordism. The Cartesian product of manifolds defines an external product on $\mathcal{U}_{*}^{G}(-)$, and a product on the spectrum. This makes $\mathcal{U}_{G}^{*}(-)$ into a multiplicative cohomology theory. In particular, $\mathcal{U}_{G}^{*}=\mathcal{U}_{G}^{*}(*)=\mathcal{U}_{-*}^{G}$ is a graded ring, and every $\mathcal{U}_{G}^{*}(X)$ is a module over this ring.

2.2 Homotopical cobordism. We said that the representing spectrum for geometric cobordism is not what we expected from the nonequivariant example. What we expected was the equivariant Thom spectrum, so let us define it and examine the theory that it does represent.

Let $B U_{G}(n)$ be the classifying space for complex $G$-bundles of complex dimension $n$. Let $\gamma_{n}$ be the universal bundle over this space. If $V$ is a complex representation of $G$, let $M U_{G}(V)$ be the Thom space of the bundle $\gamma_{|V| / 2}$. If $V \subset W$, then we have a $G$-map

$$
\sigma: \Sigma^{W-V} M U_{G}(V) \rightarrow M U_{G}(W)
$$


which comes from the classifying map for $\gamma \oplus(W-V)$. The spaces $M U_{G}(V)$, with the suspension maps $\sigma$, fit together to give a $G$-spectrum (see [12] for a general discussion of equivariant spectra). We call this spectrum $M U_{G}$, and refer to it as the unitary $G$-Thom spectrum. It represents a homology theory, called homotopical unitary bordism, written $M U_{*}^{G}(-)$, and a cohomology theory called homotopical unitary cobordism, written $M U_{G}^{*}(-)$. For example, if $n>0$, we have

$$
\widetilde{M U}_{n}^{G}(X)=\operatorname{colim}_{V}\left[S^{n} \wedge S^{V}, X \wedge M U_{G}(V)\right]_{G},
$$

where the colimit is taken over all complex representations of $G$. The other cases are defined similarly, with the $X$ on the other side for the cohomology theory. In general, these are quite different from the geometric theories.

Classification of the Whitney sum of bundles gives us a $G$-map $M U_{G}(V) \wedge$ $M U_{G}(W) \rightarrow M U_{G}(V \oplus W)$, which makes $M U_{G}$ into a ring spectrum and makes $M U_{G}^{*}(-)$ a multiplicative cohomology theory. In particular, $M U_{G}^{*}=M U_{G}^{*}(*)$ is a ring, and every $M U_{G}^{*}(X)$ is a module over it.

Homotopical cobordism enjoys a form of periodicity similar to, but weaker than, Bott periodicity. Let $V$ be a complex representation of $G$, and let $\eta_{V}$ be the class of the map $S^{|V|} \rightarrow M U_{G}(V)$ which classifies the trivial bundle $\mathbf{R}^{|V|} \rightarrow *$.

THEOREM 2.1. $\eta_{V} \in M U_{G}^{V-|V|}=M U_{|V|-V}^{G}$ is a unit of the $R O(G)$-graded ring. Multiplication by $\eta_{V}$ thus induces isomorphisms

$$
\widetilde{M U}_{m}^{G}(X) \cong \widetilde{M U}_{m+|V|}^{G}\left(\Sigma^{V} X\right)
$$

and

$$
\widetilde{M U}_{G}^{m}\left(\Sigma^{V} X\right) \cong \widetilde{M U}_{G}^{m-|V|}(X) .
$$

ProOF. $\eta_{V}^{-1}$ is given by the map $S^{V} \rightarrow M U_{G}\left(\mathbf{R}^{|V|}\right)$ which classifies the bundle $V \rightarrow *$.

This result is well known; see particularly $[\mathbf{8}, \mathbf{4}$, and $\mathbf{7}]$.

The homotopical and geometric theories are related. One relation provides a nice geometric interpretation of the homotopically defined theory. First, notice that there is a map of homology theories

$$
\Phi: U_{*}^{G}(-) \rightarrow M U_{*}^{G}(-)
$$

given by the Pontrjagin-Thom construction familiar from the nonequivariant case. (That this fails to be an isomorphism is due to the failure of transversality in this context.) Consider the following construction. Suppose that $f: M \rightarrow X$ is a unitary $G$-manifold over the $G$-space $X$, and that $V$ is a complex $G$-representation. Then $M \times D(V)$ is a unitary $G$-manifold (with boundary), and we can form the map

$$
f \times 1:(M \times D(V), M \times S(V)) \rightarrow(X \times D(V), X \times S(V)) .
$$

This defines a map

$$
\gamma_{V}: \mathcal{U}_{m}^{G}(X) \rightarrow \mathcal{U}_{m+|V|}^{G}(X \times(D(V), S(V))) .
$$

We call $\gamma_{V}$ stabilization by $V$. A similar construction can be given in the relative case, if we round corners. 
Comparing $\gamma_{V}$ to $\eta_{V}$, we can see that we have a commutative diagram

$$
\begin{array}{ccc}
\mathcal{U}_{m}^{G}(X) & \stackrel{\Phi}{\longrightarrow} & M U_{m}^{G}(X) \\
\downarrow \gamma_{V} & & \downarrow \eta_{V} \\
\mathcal{U}_{m+|V|}^{G}(X \times(D(V), S(V))) & \stackrel{\Phi}{\longrightarrow} & M U_{m+|V|}^{G}(X \times(D(V), S(V))) .
\end{array}
$$

Hence we have a map

$$
\bar{\Phi}: \operatorname{colim}_{V} U_{m+|V|}^{G}(X \times(D(V), S(V))) \rightarrow M U_{m}^{G}(X),
$$

and similarly for the relative versions. For all versions we have

THEOREM 2.2. $\bar{\Phi}$ is an isomorphism.

For the proof, see [4]. We should note that the more restrictive definition of "unitary manifold" will still make this result true, because the stabilization maps $\gamma_{V}$ effectively eliminate the difference.

This theorem leads to a nice geometric interpretation of homotopical bordism.

DEFINITION. A stable unitary $G$-manifold is the equivalence class of a unitary $G$-manifold $M$ endowed with a map $\varphi:(M, \partial M) \rightarrow(D(V), S(V))$ for some complex $G$-representation $V$. The equivalence relation is the one generated by considering $\varphi$ to be equivalent to

$$
\varphi \times 1:(M \times D(W), \partial(M \times D(W))) \rightarrow(D(V \oplus W), S(V \oplus W)),
$$

where $W$ is any complex representation of $G$.

As usual, $M \times D(W)$ has its corners rounded. The virtual dimension of $M \rightarrow$ $D(V)$ is defined to be $\operatorname{dim} M-|V|$. Stable cobordisms are defined in the obvious way. A stable manifold over a $G$-space $X$ is one with a $G$-map from the manifold part $M$ to $X$; stable cobordisms over $X$ are dealt with similarly. With careful attention to parts of boundaries, we can define relative versions of these ideas as well. Thus we can form a new homology theory by taking stable cobordism classes of stable manifolds over $X$, giving us groups $\underline{\mathcal{U}}_{*}^{G}(X)$. We call this stable unitary $G$-bordism.

These groups are easily identified with the groups

$$
\underset{V}{\operatorname{colim}} \mathcal{U}_{m+|V|}^{G}(X \times(D(V), S(V))) \text {. }
$$

In fact, a stable manifold $M \rightarrow D(V)$ over $X$ is really a manifold over the space $X \times D(V)$, and the stability equivalence relation is identical to the relation forced by the colimit maps $\gamma_{V}$. What Theorem 2.2 says then, is that homotopical bordism is identical to a stable bordism, giving us a geometric way to deal with the homotopically defined theory.

To complete the picture of homotopical bordism, we ought to say what various homotopically defined maps--such as naturality maps, Gysin maps, and Thom maps-look like in the geometric interpretation. We will do this for several maps as we need them, but here we will outline a proof scheme for verifying such interpretations. Suppose that a given homotopical construction leads to a natural map $\psi: M U_{*}^{G}(-) \rightarrow M U_{*}^{G}(-)$, and that we also have a geometric construction on manifolds that we think gives $\psi$. Suppose that this leads to a natural map $\varphi: U_{*}^{G}(-) \rightarrow \mathcal{U}_{*}^{G}(-)$. To show that our interpretation is correct, we need to check 
two things: (1) that $\varphi$ is stable, i.e., that it commutes with the maps $\gamma_{V}$, and (2) that $\varphi$ is carried to $\psi$ by $\Phi$, i.e., that $\Phi \varphi=\psi \Phi$.

In practice, we are given $\psi$ and must find $\varphi$. Happily, we can usually find $\varphi$ by assuming that transversality holds and examining the homotopical construction in this light. To actually verify that a given $\varphi$ is the correct interpretation, we use the proof scheme above, which does not need transversality. It is a pleasant exercise to work out the correspondences for various maps, and a number of these are recorded in [7].

3. Proof of Theorem A. We now show how Theorem A follows from Theorem B. The argument is due to Ib Madsen. Let $G$ be a finite group for this section.

We will need some results on equivariant transversality. Some references are $[\mathbf{1 6}, \mathbf{9}, \mathbf{1 9}$, and 7]. The sad fact is that transversality often fails in the equivariant world. One can, as in [16], develop obstructions to obtaining transversality. We quote a result of his on a sufficient condition for making these obstructions vanish.

Suppose that $M$ is a $G$-manifold and $V$ is a $G$-representation, with $f: M \rightarrow V$ a smooth $G$-map. We wish to make $f$ transverse to the origin (this is the special case we will need). Let $\tau=\tau M$ be the tangent bundle of $M$. For $H$ a subgroup of $G$, let $\hat{H}$ denote the set of irreducible real representations of $H$. If $W \in \hat{H}$, let $d_{W}=\operatorname{dim}_{\mathbf{R}} \operatorname{Hom}_{H}(W, W)$. Since $\operatorname{Hom}_{H}(W, W)$ is one of $\mathbf{R}, \mathbf{C}$, or $\mathbf{H}, d_{W}$ is one of 1,2 , or 4 .

THEOREM 3.1. Let $G$ be a finite group, $M$ a $G$-manifold, and $V$ a representation of $G$. Suppose $f: M \rightarrow V$ is a $G$-map. For $x \in M^{H}$ write $\left(\tau_{x}\right)_{H}=$ $\sum_{W \in \hat{H}} W^{a_{W}}$, and $(V)_{H}=\sum_{W \in \hat{H}} W^{b_{W}}$, where $a_{W}$ and $b_{W}$ are integers. If

$$
d_{W}\left(a_{W}-b_{W}+1\right)-1>\operatorname{dim} M^{H}-\left|V^{H}\right|
$$

for every $H \subset G$, every $x \in M^{H}$, and every nontrivial $W \in \hat{H}$, then $f$ is $G$ homotopic to a map which is transverse to the origin of $|V|$.

This is a special case of [16, II.4.13].

Let $Z$ be the complex regular representation of $G$. Let $P \subset \mathcal{U}_{G}^{*}$ be the multiplicative system generated by the class of $\mathbf{C P}(Z)$, the manifold of complex lines in $Z$ with $G$-action induced by the action of $G$ on $Z$. Since localization is exact we can form a new theory by formally inverting $P$, considering every $\mathcal{U}_{G}^{*}(X)$ to be a module over $\mathcal{U}_{G}^{*}$. In this way we get the new theory $P^{-1} \mathcal{U}_{G}^{*}(-)$. Via the ring homomorphism $\Phi$, we can also form the theory $P^{-1} M U_{G}^{*}(-)$.

THEOREM 3.2. $P^{-1} \mathcal{U}_{G}^{*}(-) \cong P^{-1} M U_{G}^{*}(-)$ via $\Phi$.

ProOF. Since both sides are $G$-cohomology theories, it suffices to show that $\Phi$ is an isomorphism on the orbits $G / H$. Since $\mathcal{U}_{G}^{*}(G / H) \cong \mathcal{U}_{H}^{*}$ and $M U_{G}^{*}(G / H) \cong$ $M U_{H}^{*}$, and $P$ is carried by these isomorphisms to a similar system, it suffices to check that $\Phi$ induces an isomorphism on the one-point space. (Actually, $P$ is carried to a slightly different system, but this can be handled by a variation of the argument which follows.) That is, we need only check that $P^{-1} \mathcal{U}_{G}^{*} \cong P^{-1} M U_{G}^{*}$.

We would like to describe an inverse to $\Phi$, call it $\Psi$. Suppose that we have a stable manifold $f: M \rightarrow D(V)$. If we could make $f$ transverse to the origin of $V$, we could form $N=f^{-1}(0)$, and it is easy to see that then $\Phi[N]=[f]$, so we would 
set $\Psi[f]=[N]$. In general this is not possible, but we will show that it is possible if we first allow ourselves to multiply $M$ by sufficiently many copies of $\operatorname{CP}(Z)$.

When do the obstructions to making a map $M \times \mathbf{C P}(Z)^{k} \rightarrow V$ transverse to the origin vanish? We check the conditions of Theorem 3.1. Let $H \subset G$ and $x \in M^{H}$. Write $\left(\tau_{x} M\right)_{H}=\sum_{W \in \hat{H}} W^{a_{W}}$, and $V_{H}=\sum_{W \in \hat{H}} W^{b_{W}}$. If $y \in\left(\mathbf{C P}(Z)^{k}\right)^{H}$, then $\left(\tau_{y} \mathbf{C P}(Z)^{k}\right)_{H} \cong Z_{H}^{k}$, so

$$
\left(\tau_{y} \mathbf{C P}(Z)^{k}\right)_{H}=\sum_{W \in \hat{H}-\mathbf{R}} W^{2 k|G: H| \cdot|W|} .
$$

Also, $\operatorname{dim}\left(\mathbf{C P}(Z)^{k}\right)^{H}=2 k|G: H|-2 k$, because the fixed-point components of CP $(Z)$ correspond to the various one-dimensional complex representations of $H$, each of which appears exactly $|G: H|$ times. Thus, the condition that we need to satisfy is

$$
d_{W}\left(2 k|G: H| \cdot|W|+a_{W}-b_{W}+1\right)-1>2 k|G: H|-2 k+\operatorname{dim} M^{H}-\left|V^{H}\right| .
$$

This is easily satisfied by taking $k$ sufficiently large.

Thus $\Psi$ is defined as follows: Given $f: M \rightarrow D(V)$, take $k$ so large that we can assume that $\tilde{f}: M \times \mathbf{C P}(Z)^{k} \rightarrow D(V)$ is transverse to the origin. Let $\Psi[f]=$ $[\mathbf{C P}(Z)]^{-k}\left[\tilde{f}^{-1}(0)\right] \in P^{-1} \mathcal{U}_{G}^{*}$. It is straightforward to check that this is the inverse to $\Phi$.

Proof of TheOrem A. Observe that $\mathbf{C P}(Z)$ maps to a unit in $K_{G}^{*}$-this is essentially the fact that the index of a projective space is 1 ; see [2]. So,

$$
\begin{aligned}
\tilde{U}_{G}^{*}(X) \otimes u_{G}^{*} K_{G}^{*} & \cong P^{-1} \tilde{U}_{G}^{*}(X) \otimes_{P^{-1} u_{G}^{*}} K_{G}^{*} \cong P^{-1} \widetilde{M U} \tilde{M}_{G}^{*}(X) \otimes_{P^{-1} M U_{G}^{*}} K_{G}^{*} \\
& \cong \widetilde{M U} U_{G}^{*}(X) \otimes_{M U_{G}^{*}} K_{G}^{*} \cong \tilde{K}_{G}^{*}(X)
\end{aligned}
$$

by Theorem B.

\section{Reductions.}

4.1 Thom classes, Euler classes, and $\mu$. We have yet to define the map $\mu$ from cobordism to $K$-theory. To do this we need a quick discussion of Thom classes.

Both $K$-theory and homotopical unitary cobordism have Thom classes for complex bundles. By this we mean that if $\xi$ is a complex $G$-bundle over a compact $G$-space $X$, such that all of the fibers have the same dimension, then there are classes $t(\xi) \in \tilde{K}_{G}^{|\xi|}(M \xi)$ and $t(\xi) \in \widetilde{M U}_{G}^{|\xi|}(M \xi)$ where $M \xi$ is the Thom space of the bundle. These Thom classes obey all of the nice properties that have become familiar in the nonequivariant case, including giving Thom isomorphisms (see [7 or 15] for more detail). The classes in $K$-theory were defined by Atiyah in [2], and are closely related to the Bott periodicity classes. The classes in cobordism are defined exactly as in the nonequivariant case: Classify $\xi$ by a map $M \xi \rightarrow M U_{G}\left(\mathbf{R}^{|\xi|}\right)$ and then interpret this map as a cobordism class.

The Thom classes in homotopical cobordism are, in a sense, universal, as in the nonequivariant case. In particular, there is a unique natural transformation of cohomology theories, $\mu: M U_{G}^{*}(-) \rightarrow K_{G}^{*}(-)$, preserving all Thom classes (see [15]). This map, which is the one used in Theorem $\mathrm{B}$, is necessarily multiplicative. The map from geometric cobordism to $K$-theory is the composite of $\mu$ with $\Phi: \mathcal{U}_{G}^{*}(-) \rightarrow$ $M U_{G}^{*}(-)$. 
Having Thom classes, we can also define Euler classes. If $\xi$ is a complex bundle over $X$, then there is a 0 -section map $s_{0}: X^{+} \rightarrow M \xi$, and we let

$$
e(\xi)=s_{0}^{*} t(\xi) \in K_{G}^{|\xi|}(X) \quad \text { or } \quad M U_{G}^{|\xi|}(X) .
$$

In particular, if $V$ is a complex representation of $G$, we may consider the trivial bundle $V \rightarrow *$ over a point; the Euler class of this bundle is an element $e_{V} \in K_{G}^{|V|}$ or $M U_{G}^{|V|}$. This class is 0 if $V$ has any trivial summands, but in general it is nonzero. We will see specific examples of this later. In passing, we mention that, as a stable cobordism class, $e_{V}$ is represented by the stable manifold $* \hookrightarrow D(V)$, inclusion of the origin.

4.2 Reduction to unitary groups.

THEOREM 4.1. Suppose that Theorem B is true for a group $G$, and that $H$ is a subgroup of $G$. Then Theorem B is true for $H$.

PROOF.

$$
M U_{H}^{*} \otimes_{M U_{G}^{*}} K_{G}^{*} \cong M U_{G}^{*}(G / H) \otimes_{M U_{G}^{*}} K_{G}^{*} \cong K_{G}^{*}(G / H) \cong K_{H}^{*} .
$$

So, for any $H$-space $X$ we have

$$
\begin{aligned}
\widetilde{M U}_{H}^{*}(X) \otimes_{M U_{H}^{*}} K_{H}^{*} & \cong \widetilde{M U}_{H}^{*}(X) \otimes_{M U_{H}^{*}}\left(M U_{H}^{*} \otimes_{M U_{G}^{*}} K_{G}^{*}\right) \\
& \cong \widetilde{M U}_{H}^{*}(X) \otimes_{M U_{G}^{*}} K_{G}^{*} \\
& \cong \widetilde{M U}{ }_{G}^{*}\left(G^{+} \wedge_{H} X\right) \otimes_{M U_{G}^{*}} K_{G}^{*} \\
& \cong \tilde{K}_{G}^{*}\left(G^{+} \wedge_{H} X\right) \cong \tilde{H}_{H}^{*}(X) .
\end{aligned}
$$

Here is the main sticking point if we wish to use something other than integer grading for Theorem $\mathrm{B}$, for example $R O(G)$ grading. Theorem 4.1 relies on the change-of-groups isomorphism, which only seems to work well with integer grading.

Recall now that any compact Lie group embeds as a closed subgroup in some $U(n)$. This gives us

COROLLARY 4.2. If Theorem B is true for the unitary groups $U(n)$, then it is true for all compact Lie groups.

4.3 The holomorphic transfer. Having reduced Theorem $\mathrm{B}$ to the case of the unitary group, we now attempt to derive that case from the known result for the torus. To help, we need a map called the holomorphic transfer, which we define.

Let $T^{n}$ be the maximal torus of $U(n)$. For convenience, let $h_{U(n)}$ be the $U(n)$ spectrum representing either homotopical unitary cobordism or $K$-theory, and let $h_{U(n)}^{*}(-)$ be the corresponding theory. Recall that

$$
\tilde{h}_{T^{n}}^{*}(X) \cong \tilde{h}_{U(n)}^{*}\left(\left(U(n) / T^{n}\right)^{+} \wedge X\right)
$$

if $X$ is a $U(n)$-space.

Take the usual unitary structure on the manifold $U(n) / T^{n}$. This will be given by a complex structure on the normal bundle $\nu$ to some embedding $U(n) / T^{n} \hookrightarrow V$, where $V$ is a complex representation of $U(n)$. This embedding gives us a collapse $\operatorname{map} c: S^{V} \rightarrow M \nu$ as usual. $\nu$ gives rise to a map $t: \Sigma^{n(n-1)} M \nu \rightarrow h_{U(n)}(V)$. In the case of $K$-theory, this is the Thom class of $\nu$ shifted into a different degree by 
Bott periodicity. In the case of cobordism, we can do the same thing using Theorem 2.1. Notice that $n(n-1)$ is the dimension of $U(n) / T^{n}$. Finally, there is a map $\Delta: M \nu \rightarrow\left(U(n) / T^{n}\right)^{+} \wedge M \nu$ induced by the diagonal map on $U(n) / T^{n}$.

Now suppose that we have an element of $\tilde{h}_{T^{n}}^{*}(X)$ represented by a $U(n)$-map

$$
g: \Sigma^{A}\left(\left(U(n) / T^{n}\right)^{+} \wedge X\right) \rightarrow h_{U(n)}(B)
$$

for some $U(n)$-representations $A$ and $B$. Then consider the composite

$$
\begin{aligned}
\Sigma^{A} \Sigma^{n(n-1)} \Sigma^{V} X & \stackrel{\Delta \circ c}{\longrightarrow} \Sigma^{A} \Sigma^{n(n-1)}\left(X \wedge\left(U(n) / T^{n}\right)^{+} \wedge M \nu\right) \\
& \stackrel{\approx}{\longrightarrow} \Sigma^{A}\left(U(n) / T^{n+} \wedge X\right) \wedge \Sigma^{n(n-1)} M \nu \\
& \stackrel{g \wedge t}{\longrightarrow} h_{U(n)}(B) \wedge h_{U(n)}(V) \\
& \longrightarrow h_{U(n)}(B \oplus V) .
\end{aligned}
$$

This defines an element of $\tilde{h}_{U(n)}^{*}(X)$, which we call $\tau[g]$. This defines a map

$$
\tau: \tilde{h}_{T^{n}}^{*}(X) \rightarrow \tilde{h}_{U(n)}^{*}(X) .
$$

We call $\tau$ the holomorphic transfer. Notice that $\tau$ lowers degree by $n(n-1)$.

This is a simple case of the sort of construction discussed in [11] (see particularly his Remark 4.8). $\tau$ is essentially a transfer for bundles of the form $U(n) / T^{n} \times X \rightarrow$ $X$. It is a map of $h_{U(n)}^{*}$-modules. If we let $\xi: \tilde{h}_{U(n)}^{*}(X) \rightarrow \tilde{h}_{T^{n}}^{*}(X)$ be the forgetful map, then, as with any transfer, $\tau \circ \xi$ is just multiplication by the Euler class of the transfer, $\chi=\tau \xi(1) \in h_{U(n)}^{-n(n-1)}$. Since $\tau$ was defined by means of the Thom class, $\mu$ will preserve it and $\chi$.

We can identify $\tau$ more precisely in these two theories.

PROPOSITION 4.3. $\tau: \tilde{K}_{T^{n}}^{*}(-) \rightarrow \tilde{K}_{U(n)}^{*}(-)$ is, up to periodicity, the holomorphic transfer of [2 and 3]. In particular, $\chi$ is a unit of the ring $K_{U(n)}^{*}$.

The argument is given in [14] (see the remark after his 5.2), as noted in [11]. It comes down to the fact that $\tau$ is defined in terms of the topological index, while Atiyah's holomorphic transfer is defined in terms of the analytical index. The Atiyah-Singer Index Theorem serves to identify them. That $\chi$ is a unit follows from [2], which shows that $\chi$ is a Bott class. This result justifies our calling $\tau$ the holomorphic transfer.

Proposition 4.4. $\tau: M U_{T^{n}}^{*} \rightarrow M U_{U_{(n)}^{*}}^{*}$ is given geometrically as the map which takes a $T^{n}$-manifold $M$ to the $U(n)$-manifold $U(n) \times_{T^{n}} M$. In particular, $\chi$ is the class of $U(n) / T^{n}$ in $M U_{U(n)}^{-n(n-1)}$.

The proof follows the outline given at the end of $\S 2$.

Proposition 4.3 suggests the following trick. Let $D \subset M U_{U(n)}^{*}$ be the multiplicative set of elements which get sent to units in $K_{U(n)}^{*}$ by $\mu$. Since localization is exact, we can form a new cohomology theory $D^{-1} M U_{U(n)}^{*}(-)$. This new theory is at least as useful to us as the old one because of the easy observation

PROPOSITION 4.5.

$$
D^{-1} \widetilde{M U}_{U(n)}^{*}(X) \otimes_{D^{-1} M U_{U(n)}^{*}} K_{U(n)}^{*} \cong \widetilde{M U}_{U(n)}^{*}(X) \otimes_{M U_{U(n)}^{*}} K_{U(n)}^{*} .
$$

The new theory is better than the old one in the following way. 
PROPOSITION 4.6. $\xi: D^{-1} \widetilde{M U}_{U(n)}^{*}(X) \rightarrow D^{-1} \widetilde{M U}_{T^{n}}^{*}(X)$ is a naturally split monomorphism.

ProOF. A splitting is given by $\chi^{-1} \tau$, since $\chi \in D$ by Proposition 4.3, and $\tau \xi(x)=\chi x \forall x \in \widetilde{M U}_{U(n)}^{*}(X)$.

This gives us our next reduction of Theorem B.

THEOREM 4.7. If $M U_{T^{n}}^{*} \otimes_{M U_{U(n)}^{*}} K_{U(n)}^{*} \cong K_{T^{n}}^{*}$, then Theorem B is true for $U(n)$.

PROOF. Consider the diagram

$$
\begin{array}{ccccc}
0 \rightarrow & D^{-1} \widetilde{M U}_{U(n)}^{*}(X) \otimes_{D^{-1} M U_{U(n)}^{*}}^{*} K_{U(n)}^{*} & \stackrel{\xi}{\rightarrow} & D^{-1} \widetilde{M U}_{T^{n}}^{*}(X) \otimes_{D^{-1} M U_{U(n)}^{*}} K_{U(n)}^{*} \\
& \downarrow \mu & & \downarrow \mu \\
0 & \tilde{K}_{U(n)}^{*}(X) & \stackrel{\xi}{\longrightarrow} & \tilde{K}_{T^{n}}^{*}(X) .
\end{array}
$$

The maps $\xi$ are monomorphisms with compatible splittings. The map $\mu$ on the left is the one we wish to show is an isomorphism. A simple diagram chase shows that it suffices to show that the $\mu$ on the right is an isomorphism. With our assumption it is:

$$
\begin{aligned}
& D^{-1} \widetilde{M U}_{T^{n}}^{*}(X) \otimes_{D^{-1} M U_{U(n)}^{*}} K_{U(n)}^{*} \cong \widetilde{M U}_{T^{n}}^{*}(X) \otimes_{M U_{U(n)}^{*}} K_{U(n)}^{*} \\
& \cong \widetilde{M U}_{T^{n}}^{*}(X) \otimes_{M U_{T^{n}}^{*}}\left(M U_{T^{n}}^{*} \otimes_{M U_{U(n)}^{*}} K_{U(n)}^{*}\right) \\
& \cong \widetilde{M U}_{T^{n}}^{*}(X) \otimes_{M U_{T^{n}}^{*}} K_{T^{n}}^{*} \cong \tilde{K}_{T^{n}}^{*}(X) \text {. }
\end{aligned}
$$

The last isomorphism is Okonek's result for abelian groups [15].

\section{The rest of the proof.}

5.1 Euler classes again. What remains to be proved is the isomorphism

$$
M U_{T^{n}}^{*} \otimes_{M U_{U}^{*}(n)} K_{U(n)}^{*} \cong K_{T^{n}}^{*}
$$

As usual, we may replace $M U_{U(n)}^{*}$ with $D^{-1} M U_{U(n)}^{*}$ and so on. Equation (*) is a relation between four rings. We know the structure of two of them. Namely, $K_{G}^{*}$ is $R(G)$ in even degrees, and 0 in odd degrees. The multiplication is specified by the fact that there is a unit in degree 2 . Therefore, studying $K_{G}^{*}$ is just like studying $R(G)$. We know the structures of $R\left(T^{n}\right)$ and $R(U(n)$ ) quite well (see, e.g., [1]). In particular, $R\left(T^{n}\right)$ is a free $R(U(n))$-module on $n$ ! basis elements. We can specify those basis elements as follows. Let $V_{1}, \ldots, V_{n}$ be the $n$ fundamental onedimensional complex representations of $T^{n}$ given by the projections of $T^{n}=S^{1} \times$ $\cdots \times S^{1}$ onto its factors. Let $e_{1}, \ldots, e_{n}$ be the Euler classes of these representations. Then a basis for $R\left(T^{n}\right)$ as an $R(U(n))$-module is given by $\left\{e_{2}^{i_{2}} \cdots e_{n}^{i_{n}} \mid 0 \leq i_{j} \leq\right.$ $j-1\}$ (combine 6.20 of [1] with IV.3.28 of [10]). The same is then true of the rings $K_{T^{n}}^{*}$ and $K_{U(n)}^{*}$. More generally, $R\left(T^{n}\right)$ is a free $R\left(U(k) \times T^{n-k}\right)$-module with basis $\left\{e_{2}^{i_{2}} \cdots e_{k}^{i_{k}} \mid 0 \leq i_{j} \leq j-1\right\}$. 
Since the Euler classes already exist in homotopical cobordism, we see that we have

Proposition 5.1. $\mu: M U_{T^{n}}^{*} \otimes_{M U_{U(n)}^{*}} K_{U(n)}^{*} \rightarrow K_{T^{n}}^{*}$ is a split epimorphism of $K_{U(n)}^{*}$-modules.

Notice that an explicit splitting is given by taking the basis element $e_{2}^{i_{2}} \cdots e_{n}^{i_{n}}$ in $K_{T^{n}}^{*}$ to the element of the same name in $M U_{T^{n}}^{*}$. To show that $\mu$ is a monomorphism, we need to show that this splitting is an epimorphism. We shall show, in fact, that these elements generate $D^{-1} M U_{T^{n}}^{*}$ as a $D^{-1} M U_{U(n)}^{*}$-module.

5.2 Symmetry. Recall that the Weyl group of $U(n)$ is $N\left(T^{n}\right) / T^{n} \cong \Sigma_{n}$, the symmetric group on $n$ letters. To be precise, the isomorphism is specified by considering the action of $N\left(T^{n}\right) / T^{n}$ on the left on $T^{n}$ by conjugation. This action permutes the factors, defining the desired map into $\Sigma_{n}$. We henceforth identify $N\left(T^{n}\right) / T^{n}$ and $\Sigma_{n}$.

Consider now the right action of $\Sigma_{n}$ on $T^{n}$ given by $t \sigma=\sigma^{-1} t$. This induces a left action of $\Sigma_{n}$ on $R\left(T^{n}\right)$, which permutes the representations $V_{1}, \ldots, V_{n}$ in the expected way. Hence it permutes the Euler classes by $\sigma e_{k}=e_{\sigma(k)}$.

$\Sigma_{n}$ acts by multiplication on the right on $U(n) / T^{n}$, which is an action by $U(n)$ maps. This produces a left action on $M U_{T^{n}}^{*} \cong M U_{U(n)}^{*}\left(U(n) / T^{n}\right)$, by $M U_{U(n)^{-}}^{*}$ module maps, which in turn gives us an action on $D^{-1} M U_{T^{n}}^{*}(-)$. Alternatively, this action is given by twisting the action of $T^{n}$ on a manifold by the right action of $\Sigma_{n}$ on $T^{n}$. Again, the action on the Euler classes is as expected.

Let $\Sigma_{k} \subset \Sigma_{n}$ be the subgroup of elements that permute only the first $k$ letters. Define a sequence of rings by

$$
R_{k}=\left(D^{-1} M U_{T^{n}}^{*}\right)^{\Sigma_{k}}
$$

We will show two results.

PrOpOSITION 5.2. $R_{k-1}$ is generated as an $R_{k}$-module by $1, e_{k}, \ldots, e_{k}^{k-1}$.

Proposition 5.3. $R_{n} \cong D^{-1} M U_{U(n)}^{*}$ via $\xi$.

5.3 Families and Proposition 5.3. To prove these results we need to bring in the idea of a family of subgroups. Recall that a family of subgroups of $G$ is a collection of subgroups that is closed under conjugation and taking of subgroups. The only families that we will need are $A$, the family of all subgroups, and $P$, the family of proper subgroups $(A-G)$. [5] introduced the idea of a manifold with restricted isotropy. In our case, we are interested in so-called $(A, P)-G$-manifolds, which are $G$-manifolds with no fixed-points on their boundaries. That is, the isotropy subgroups of the boundaries are restricted to be in $P$, while there is no restriction in the interior.

As in [5], we can define cobordism of $(A, P)$-manifolds and define cobordism theories using them. Similarly, we can define stable $(A, P)$-manifolds and cobordisms. In particular, we can look at the ring of stable cobordism classes of stable unitary $(\mathcal{A}, P)-T^{n}$-manifolds, which we call $M U_{T^{n}}^{*}[\mathcal{A}, P]$.

Since a manifold with no boundary is certainly an $(A, P)$-manifold, there is a map

$$
F: M U_{T^{n}}^{*} \rightarrow M U_{T^{n}}^{*}[A, P]
$$


The remarkable fact about this map is:

THEOREM 5.4 (LÖFFLER). F is a monomorphism.

For the proof see [13]. It is well known from [5] that an $(\mathcal{A}, P)$-manifold is determined, up to cobordism, by a neighborhood of its submanifold of fixed-points. In particular, it is determined by its fixed-points and the normal bundle to their inclusion. Theorem 5.4 allows us to say the same thing about cobordism classes of $T^{n}$-manifolds without boundary. Here is an example of its use:

PrOOF OF Proposition 5.3. We already know that $\xi: D^{-1} M U_{U(n)}^{*} \rightarrow$ $D^{-1} M U_{T^{n}}^{*}$ is a monomorphism, and its image is clearly contained in $R_{n}$. We must show that it maps onto $R_{n}$.

Consider $F \xi \tau \xi(1)=F \xi(\chi)=F\left[U(n) / T^{n}\right]$. As a $T^{n}$-manifold, $U(n) / T^{n}$ has $n$ ! discrete fixed-points, namely the set $N\left(T^{n}\right) / T^{n}=\Sigma_{n}$. Since we can restrict our attention to a neighborhood of these fixed-points, we can write

$$
F\left[U(n) / T^{n}\right]=\sum_{\sigma \in \Sigma_{n}} d_{\sigma}
$$

where $d_{\sigma}$ is, up to sign, the class of the disc of the tangent $T^{n}$-representation at $\sigma \in U(n) / T^{n}$.

More generally, let $M$ be any $T^{n}$-manifold, and consider

$$
F \xi \tau[M]=F\left[U(n) \times_{T^{n}} M\right] .
$$

We notice that

$$
\left(U(n) \times_{T^{n}} M\right)^{T^{n}} \subset N\left(T^{n}\right) \times_{T^{n}} M,
$$

and that this latter space consists of $n$ ! copies of $M$, sitting over $\Sigma_{n}$, with the $T^{n}$ action on each twisted by the action of the corresponding element of $\Sigma_{n}$. Since a neighborhood of one of these copies of $M$ in $U(n) \times_{T^{n}} M$ looks like the product of $M$ (with twisted action) and a neighborhood of the corresponding $\sigma \in \Sigma_{n} \subset U(n) / T^{n}$, we see that

$$
F \xi \tau[M]=\sum_{\sigma \in \Sigma_{n}} d_{\sigma} \sigma F[M]
$$

This passes to stable manifolds, giving us

$$
F \xi \tau(m)=\sum_{\sigma \in \Sigma_{n}} d_{\sigma} \sigma F(m) \quad \forall m \in D^{-1} M U_{T^{n}}^{*}
$$

Now notice that if $m \in R_{n}$, then $\sigma F(m)=F(m)$ for every $\sigma \in \Sigma_{n}$, so that

$$
F \xi \tau(m)=\left(\sum_{\sigma \in \Sigma_{n}} d_{\sigma}\right) F(m)=(F \xi \chi) F(m)=F(\chi m) .
$$

Since $F$ is injective, this says that $\xi \tau(m)=\chi m$, or that $m=\xi\left(\chi^{-1} \tau(m)\right)$. Hence $m$ is in the image of $\xi$, and $\xi$ maps onto $R_{n}$.

5.4 The idea of the proof of 5.2. In order to prove Proposition 5.2, we need to show that any $m \in R_{k-1}$ can be written as

$$
m=\sum_{i=0}^{k-1} a_{i} e_{k}^{i}
$$


for some elements $a_{i} \in R_{k}$. This is a single equation in the $k$ unknowns $a_{i}$, but we can convert it into $k$ equations by applying the transpositions $(j k) \in \Sigma_{k}$. This gives us the system of equations

$$
(j k) m=\sum_{i=0}^{k-1} a_{i} e_{j}^{i}, \quad 1 \leq j \leq k,
$$

since the $a_{i}$ are fixed by $\Sigma_{k}$.

We can solve this system formally using Cramer's rule. This expresses the $a_{i}$ as quotients of determinants. The question is: Does this division make sense in the rings in question? We will show that it does by constructing an element similar to the denominator, showing that it is invertible, and then showing that that implies that the division is possible. Our next task is to set up the tools to do the construction.

$5.5 A$ representation. Consider $W_{k}=u(k)$, the adjoint representation of $U(k)$. This is a real representation of dimension $k^{2}$. In fact, it is better than that:

PROPOSITION 5.5. $W_{k}$ is a $\operatorname{Spin}^{c}$ representation of $U(k)$.

PROOF. (This argument was shown to me by Dick Lashof.) Recall that

$$
\operatorname{Spin}^{c}\left(k^{2}\right)=\operatorname{Spin}\left(k^{2}\right) \times \mathbf{z}_{2} U(1),
$$

and that $U(k)=S U(k) \times \mathbf{z}_{k} U(1)$. In the last statement, $U(1)$ is the center of $U(k)$, and $\mathbf{Z}_{k}$ is the center of $S U(k)$.

$W_{k}$ has a trivial summand corresponding to the center of $U(k)$; write $W_{k}=$ $W_{k}^{\prime} \oplus \mathbf{R} . W_{k}^{\prime}$ is the representation given by $\rho$, the composite along the bottom in the diagram

$$
U(k) \rightarrow U(k) / U(1)=S U(k) / \mathbf{Z}_{k} \longrightarrow s u(k)
$$

Consider $s u(k)$ :

$$
S U(k) \stackrel{\sigma}{\stackrel{\operatorname{Spin}\left(k^{2}-1\right)}{\downarrow} \stackrel{\downarrow}{\longrightarrow} S O\left(k^{2}-1\right)=\operatorname{Spin}\left(k^{2}-1\right) / \mathbf{Z}_{2} .}
$$

$\sigma$ exists because $S U(k)$ is simply connected. $\sigma\left(\mathbf{Z}_{k}\right) \subset \mathbf{Z}_{2}$ since $s u(k)\left(\mathbf{Z}_{k}\right)=\{I\}$.

If $k$ is odd, then $\sigma\left(\mathbf{Z}_{k}\right)=\{I\}$, so $\sigma$ factors to give $\bar{\sigma}: S U(k) / \mathbf{Z}_{k} \rightarrow \operatorname{Spin}\left(k^{2}-1\right)$, which, composed with $U(k) \rightarrow S U(k) / \mathbf{Z}_{k}$, gives a lift of $\rho$. In this case $W_{k}$ is actually a Spin representation, and hence a $\operatorname{Spin}^{c}$ representation.

If $k$ is even, notice that $\sigma\left(2 \mathbf{Z}_{k}\right)=\{I\}$, so we have $\bar{\sigma}: S U(k) / 2 \mathbf{Z}_{k} \rightarrow \operatorname{Spin}\left(k^{2}-1\right)$. We lift $\rho$ by the composite

$$
\begin{aligned}
U(k)=S U(k) \times \mathbf{z}_{k} U(1) & \stackrel{(q, l)}{\longrightarrow} S U(k) / 2 \mathbf{Z}_{k} \times \mathbf{z}_{2} U(1) \\
& \stackrel{(\bar{\sigma}, 1)}{\longrightarrow} \operatorname{Spin}\left(k^{2}-1\right) \times \mathbf{z}_{2} U(1)=\operatorname{Spin}^{c}\left(k^{2}-1\right),
\end{aligned}
$$

where $q$ is the quotient map, and $l(\lambda)=\lambda^{k / 2}$ for $\lambda \in U(1)$.

$W_{k}$ is of interest to us mainly because of the following fact. 
PROPOSITION 5.6. There is an embedding of $U(k) / T^{k}$ in $W_{k}$ with normal bundle $U(k)$-isomorphic to $U(k) / T^{k} \times \mathbf{R}^{k}$.

PROOF. To get an embedding, we need a point in $W_{k}$ with isotropy exactly $T^{k}$. We can appeal to the theory of principal orbit types, which says that the points with isotropy conjugate to $T^{k}$ are actually dense. Or, we can be a little more direct, and notice that any point of $W_{k}$ will do if the closure of the one-parameter subgroup of $U(k)$ it determines is $T^{k}$. For, if $w$ is a point with this property, then its isotropy group will be the centralizer of $T^{k}$, which is just $T^{k}$ itself. Since $T^{k}$ is monogenic, such points exist.

Suppose then that we have chosen an embedding $i: U(k) / T^{k} \hookrightarrow W_{k}$. The normal bundle to $i$ must be of the form $U(k) \times_{T^{k}} V$ for some representation $V$ of $T^{k}$ (all bundles over $U(k) / T^{k}$ must be of this form; see [17]). The tangent bundle of $U(k) / T^{k}$ is $U(k) \times_{T^{k}} V^{\prime}$, where $V^{\prime}$ is the tangent representation at $I T^{k} \in U(k) / T^{k}$. As a representation of $T^{k}, V^{\prime}$ has no trivial summands. By virtue of the embedding, we must have $V \oplus V^{\prime} \cong W_{k}$ as representations of $T^{k}$, which means that we must have $V \cong W_{k}^{T^{k}}$. In other words, $V$ is trivial, and the normal bundle has the form stated.

We use this embedding to give $U(k) / T^{k} \times U(k) / T^{k}$, and hence

$$
\left(U(k) \times T^{n-k} / T^{n}\right) \times\left(U(k) \times T^{n-k} / T^{n}\right),
$$

a nonstandard unitary structure. We give $W_{k} \oplus W_{k}$ the complex structure making it $W_{k} \otimes_{\mathbf{R}}$ C. Then the product embedding $U(k) / T^{k} \times U(k) / T^{k} \hookrightarrow W_{k} \oplus W_{k}$ has normal bundle $U(k) / T^{k} \times U(k) / T^{k} \times\left(\mathbf{R}^{k} \oplus \mathbf{R}^{k}\right)$, which we give the complex structure making it $U(k) / T^{k} \times U(k) / T^{k} \times \mathbf{C}^{k}$.

For use below, we can determine the tangent space at $I T^{k} \times I T^{k} \in U(k) / T^{k} \times$ $U(k) / T^{k}$ as a complex representation of $T^{k}$, using this nonstandard structure. Since the normal representation to the embedding consists of all of the trivial summands of $W_{k} \otimes_{\mathbf{R}} \mathbf{C}$, the tangent space must be

$$
\left(W_{k} \otimes_{\mathbf{R}} \mathbf{C}\right)_{T^{k}} \cong u(k)_{T^{k}} \otimes_{\mathbf{R}} \mathbf{C} \cong \bigoplus_{\substack{i \neq j \\ i, j \leq k}} V_{i} \otimes \bar{V}_{j},
$$

where $V_{i}$ is the fundamental representation of $T^{k}$ defined earlier, and $\bar{V}_{j}$ indicates the complex conjugate representation.

The representation $W_{k}$ gives rise to several maps. First, the embedding $U(k) / T^{k}$ $\hookrightarrow W_{k}$ gives a collapse map $c_{k}: S^{W_{k}} \rightarrow \Sigma^{k} U(k) / T^{k^{+}}$, and hence a map

$$
\begin{aligned}
c_{k}^{*}: M U_{T^{n}}^{m} & \cong M U_{U(k) \times T^{n-k}}^{m}\left(U(k) \times T^{n-k} / T^{n}\right) \\
& =M U_{U(k) \times T^{n-k}}^{m}\left(U(k) / T^{k}\right) \quad\left(\text { trivial } T^{n-k} \text {-action }\right) \\
& \cong \widetilde{M U} U_{U(k) \times T^{n-k}}^{m+k}\left(\Sigma^{k} U(k) / T^{k^{+}}\right) \\
& \rightarrow \widetilde{M U}_{U(k) \times T^{n-k}}^{m+k}\left(S^{W_{k}}\right) .
\end{aligned}
$$

Next, the complex structure on $W_{k} \oplus W_{k}$ gives an isomorphism

$$
\eta_{k}: \widetilde{M U}_{U(k) \times T^{n-k}}^{m}\left(S^{W_{k} \oplus W_{k}}\right) \cong M U_{U(k) \times T^{n-k}}^{m-2 k^{2}}
$$

via Theorem 2.1. 
Finally, we let

$$
\xi_{k}: M U_{U(k) \times T^{n-k}}^{*} \rightarrow M U_{T^{n}}^{*}
$$

be the forgetful map.

5.6 A calculation. Our most important construction can be described simply as follows. Suppose that $\alpha$ and $\beta$ are two elements of $M U_{T^{n}}^{*}$. Then we can form the element

$$
\xi_{k} \eta_{k}\left(c_{k}^{*} \alpha \cdot c_{k}^{*} \beta\right) \in M U_{T^{n}}^{*}
$$

We wish to calculate the result in terms of $\alpha$ and $\beta$. What we do is calculate what $F$ does to it, i.e., we will look at the fixed-points.

The first thing we can do is interpret the whole construction geometrically. We state the result without proof; the program at the end of $\S 2$ can be used to verify it. Suppose that $\alpha$ is represented by $M$ and $\beta$ by $N$, with the unitary structures given by embeddings $M \hookrightarrow Z$ and $N \hookrightarrow Z^{\prime}$. (We ignore the other part of the structure of a stable manifold, the map into the disk of a complex representation. This will just pass through the construction in an uninteresting way.) We may assume, by stabilizing, that the representations $Z$ and $Z^{\prime}$ are actually complex $\oplus$ trivial representations of $U(k) \times T^{n-k} . \xi_{k} \eta_{k}\left(c_{k}^{*} \alpha \cdot c_{k}^{*} \beta\right)$ is then represented by the manifold

$$
\left[\left(U(k) \times T^{n-k}\right) \times_{T^{n}} M\right] \times\left[\left(U(k) \times T^{n-k}\right) \times_{T^{n}} N\right] \cong\left(U(k) \times_{T^{k}} M\right) \times\left(U(k) \times_{T^{k}} N\right),
$$

with the unitary structure given by the embedding into $W_{k} \oplus W_{k} \oplus Z \oplus Z^{\prime}$ induced by the given unitary structures on $M$ and $N$ and the embedding of $U(k) / T^{k}$ in $W_{k}$ that we constructed.

The $T^{n}$-fixed-point set is contained in

$$
\left(N\left(T^{k}\right) \times_{T^{k}} M\right) \times\left(N\left(T^{k}\right) \times_{T^{k}} N\right) .
$$

This set consists of copies of $M \times N$, one for each element of $\Sigma_{k} \times \Sigma_{k}$, with the action of $T^{n}$ twisted on each copy according to the corresponding pair of permutations. The normal bundle to the inclusion of a copy of $M \times N$ is the trivial bundle with fiber the tangent representation at $(\sigma, \tau) \in \Sigma_{k} \times \Sigma_{k} \subset U(k) / T^{k} \times U(k) / T^{k}$. However, our nonstandard unitary structure on $U(k) / T^{k} \times U(k) / T^{k}$ induces a nonstandard complex structure on this representation, which was determined above.

Finally, there is a question of signs. This can be resolved by looking at the embedding

$$
\Sigma_{k} \times \Sigma_{k}=N\left(T^{k}\right) / T^{k} \times N\left(T^{k}\right) / T^{k} \hookrightarrow W_{K}^{T^{k}} \otimes_{\mathbf{R}} \mathbf{C}
$$

and how the normal bundle is sent in by this map. At $(e, e)$ the normal fiber is identified with the representation, and the fiber over $(\sigma, \tau)$ gets twisted by the action of those permutations; we see that the sign which must be attached is $(-1)^{|\sigma|+|\tau|}$, where $|\sigma|$ is 0 or 1 , as $\sigma$ is an even or odd permutation.

Putting this all together, we have our calculation.

Calculation 5.7 .

$$
F \xi_{k} \eta_{k}\left(c_{k}^{*} \alpha \cdot c_{k}^{*} \beta\right)=\delta_{k}\left(\sum_{\sigma \in \Sigma_{k}}(-1)^{|\sigma|} \sigma \alpha\right)\left(\sum_{\sigma \in \Sigma_{k}}(-1)^{|\sigma|} \sigma \beta\right)
$$


where

$$
\delta_{k}=\left[D\left(\bigoplus_{\substack{i \neq j \\ i, j \leq k}} V_{i} \otimes \bar{V}_{j}\right)\right]=\prod_{\substack{i \neq j \\ i, j \leq k}}\left[D\left(V_{i} \otimes \bar{V}_{j}\right)\right] .
$$

5.7 THE PROOF OF PROPOSITION 5.2. Let

$$
\varepsilon_{k}=c_{k}^{*}\left(e_{2} e_{3}^{2} \cdots e_{k}^{k-1}\right) \in M U_{U(k) \times T^{n-k}}^{k^{2}-W_{k}} .
$$

(We will work with $R O(G)$-grading in this section.)

LEMMA 5.8. $\mu\left(\varepsilon_{k}\right) \in K_{U(k) \times T^{n-k}}^{*}$ is a unit of the ring.

PROOF. First, notice that, by Calculation 5.7,

$$
F \xi_{k} \eta_{k} c_{k}^{*}\left(e_{2}^{i_{2}} \cdots e_{k}^{i_{k}}\right)^{2}=\delta_{k}\left(\sum_{\sigma \in \Sigma_{k}}(-1)^{|\sigma|} \sigma\left(e_{2}^{i_{2}} \cdots e_{k}^{i_{k}}\right)\right)^{2} .
$$

This is 0 if any $i_{r}=i_{s}$, for then the terms in the sum will cancel in pairs. If we now require that $0 \leq i_{j} \leq j-1$, then the result will be 0 except when $i_{j}=j-1$ for each $j$. Since $F$ is injective, this vanishing remains true without it. Since the $K$-theory versions of $\xi_{k}$ and $\eta_{k}$ are injective, it follows that $\mu\left(c_{k}^{*}\left(e_{2}^{i_{2}} \cdots e_{k}^{i_{k}}\right)\right)^{2}=0$ in $K$-theory unless $i_{j}=j-1$ for all $j$.

Now $\mu\left(c_{k}^{*}\left(e_{2}^{i_{2}} \cdots e_{k}^{i_{k}}\right)\right) \in K_{U(k) \times T^{n-k}}^{N-W_{k}}$ for some integer $N$ with $N-\left|W_{k}\right|$ even. Since $W_{k}$ is $\operatorname{Spin}^{c}$ by 5.5 , this group is a copy of $R O\left(U(k) \times T^{n-k}\right)$, and there are no zero-divisors in it. Thus it must be that $\mu\left(c_{k}^{*}\left(e_{2}^{i_{2}} \cdots e_{k}^{i_{k}}\right)\right)=0$ unless $i_{j}=j-1$ for all $j$.

Now if we look at the definition of $c_{k}^{*}$, considered as a map $K_{T^{n}}^{\alpha} \rightarrow K_{U(k) \times T^{n-k}}^{\alpha+k-W_{k}}$, it is easy to see that it is just evaluation at a fundamental class of the manifold $U(k) / T^{k}$. The machinery of equivariant Poincaré duality now tells us that it is an epimorphism (see [20 or 12]). But we have just shown that it kills all but one of the basis elements of $R\left(T^{n}\right)$ as a module over $R\left(U(k) \times T^{n-k}\right)$, hence it must carry that last basis element to a generator, i.e., a unit of the ring. That is, $\mu\left(\varepsilon_{k}\right)$ is a unit.

LEMMA 5.9. If $b \in D^{-1} M U_{T^{n}}^{*}$ is an element in integer grading for which $\mu(b)$ is a unit, then $b$ is a unit.

ProOF. Let $\bar{b}$ be the product of the elements in the $\Sigma_{n}$-orbit of $b$. Then $\bar{b} \in D^{-1} M U_{U(n)}^{*}$ by 5.3. $\mu(\bar{b})$ is a unit since $\mu(b)$ is, but $\mu(\bar{b}) \in K_{U(n)}^{*}$. Write $\bar{b}=m / d$ where $m$ and $d$ are in $M U_{U(n)}^{*}$ and $d \in D$. Then $\mu(m)=\mu(d) \mu(\bar{b})$ is a unit, and $m \in D$. Hence $\bar{b}$ is invertible in $D^{-1} M U_{U(n)}^{*}$. Since $b$ divides $\bar{b}$ in $D^{-1} M U_{T^{n}}^{*}$, it must also be invertible.

PROOF OF PROPOSITION 5.2. Notice that $\xi_{k}$ maps $D^{-1} M U_{U(k) \times T^{n-k}}^{*}$ into $R_{k}$ (we could show, as in Proposition 5.3, that it is actually onto). By Lemma 5.8 and the fact that $\eta_{k}$ is multiplication by a unit, $\mu\left(\xi_{k} \eta_{k} \varepsilon_{k}^{2}\right)$ is a unit. Since $\xi_{k} \eta_{k} \varepsilon_{k}^{2}$ lies in integer grading, Lemma 5.9 shows that it is invertible in $D^{-1} M U_{T^{n}}^{*}$. We claim that if $m \in R_{k-1}$, then it can be written as

$$
m=\frac{1}{\xi_{k} \eta_{k} \varepsilon_{k}^{2}} \sum_{i=0}^{k-1} \xi_{k} \eta_{k}\left(\varepsilon_{k} m_{i}\right) e_{k}^{i}
$$


where $m_{i}=c_{k}^{*}\left(e_{2} \cdots e_{i}^{i-1}(i k) m e_{i+2}^{i+1} \cdots e_{k}^{k-1}\right)$. If true, then we are done, since the coefficients certainly lie in $R_{k}$.

Apply $F$ and use Calculation 5.7 to examine the coefficients:

$$
F \xi_{k} \eta_{k} \varepsilon_{k}^{2}=\delta_{k} \Delta_{k}^{2}
$$

and

$$
F \xi_{k} \eta_{k}\left(\varepsilon_{k} m_{i}\right)=\delta_{k} \Delta_{k} \sum_{\sigma \in \Sigma_{k}}(-1)^{|\sigma|} \sigma m_{i}
$$

where $\Delta_{k}=\sum_{\sigma \in \Sigma_{k}}(-1)^{|\sigma|} \sigma\left(e_{2} e_{3}^{2} \cdots e_{k}^{k-1}\right)$. Hence the coefficient of $e_{k}^{i}$ is, after applying $F$,

$$
\frac{1}{\Delta_{k}} \sum_{\sigma \in \Sigma_{k}}(-1)^{|\sigma|} \sigma m_{i}
$$

which is the quotient of determinants:

$$
\frac{\left|\begin{array}{lllllllll}
1 & e_{1} & e_{1}^{2} & \cdots & e_{1}^{i-1} & (1 k) m & e_{1}^{i+1} & \cdots & e_{1}^{k-1} \\
1 & e_{2} & e_{2}^{2} & \cdots & e_{2}^{i-1} & (2 k) m & e_{2}^{i+1} & \cdots & e_{2}^{k-1} \\
1 & e_{k} & e_{k}^{2} & \cdots & e_{k}^{i-1} & m & e_{k}^{i+1} & \cdots & e_{k}^{k-1}
\end{array}\right|}{\mid}
$$

By Cramer's rule, this is the solution to the system of equations

$$
(j k) m=\sum_{i=0}^{k-1} a_{i} e_{j}^{i}, \quad 1 \leq j \leq k,
$$

and the last of these is the equation we wish to hold true. Thus we have the equality when $F$ is applied, hence without it, and we are done.

CoRollary 5.10. $M U_{T^{n}}^{*} \otimes_{M U_{U(n)}^{*}} K_{U(n)}^{*} \cong K_{T^{n}}^{*}$.

PROOF. Propositions 5.2 and 5.3 show that the set $\left\{e_{2}^{i_{2}} \cdots e_{n}^{i_{n}} \mid 0 \leq i_{j} \leq j-1\right\}$ generates $D^{-1} M U_{T^{n}}^{*}$ as a $D^{-1} M U_{U(n)}^{*}$-module. Since the elements of the same name form a basis for $K_{T^{n}}$ as a module over $K_{U(n)}^{*}$, the result follows.

This completes the proof of Theorem B.

\section{REFERENCES}

1. J. F. Adams, Lectures on Lie groups, University of Chicago Press, Chicago, 1982.

2. M. F. Atiyah, Bott periodicity and the index of elliptic operators, Quart. J. Math. Oxford Ser. (2) 19 (1968), 113-140.

3. M. F. Atiyah and G. B. Segal, Equivariant K-theory and completion, J. Differential Geom. 3 (1969), 1-18.

4. T. Bröcker and E. C. Hook, Stable equivariant bordism, Math. Z. 129 (1972), $269-277$.

5. P. E. Conner and E. E. Floyd, Differentiable periodic maps, Springer-Verlag, Berlin and New York, 1964.

6. $\ldots$, The relation of cobordism to $K$-theories, Lecture Notes in Math., vol. 28, Springer-Verlag, Berlin and New York, 1966.

7. S. R. Costenoble, Equivariant cobordism and K-theory, Ph.D. Thesis, Univ. of Chicago, 1985. 
8. T. tom Dieck, Bordism of $G$-manifolds and integrality theorems, Topology 9 (1970), 345-358.

9. H. Hauschild, Äquivariante Transversalität und äquivariante Bordismentheorien, Arch. Math. 26 (1975), 536-546.

10. M. Karoubi, K-theory, Springer-Verlag, Berlin and New York, 1978.

11. L. G. Lewis, Jr., The uniqueness of bundle transfers, Math. Proc. Cambridge Philos. Soc. 93 (1983), 87-111.

12. L. G. Lewis, Jr., J. P. May and M. Steinberger, Equivariant stable homotopy theory, Lecture Notes in Math., vol. 1213, Springer-Verlag, Berlin and New York, 1986.

13. P. Löffler, Bordismengruppen unitärer Torusmannigfaltigkeiten, Manuscripta Math. 12 (1974), 307-327.

14. G. Nishida, The transfer homomorphism in equivariant generalized cohomology theories, J. Math. Kyoto Univ. 18-3 (1978), 435-451.

15. C. Okonek, Der Conner-Floyd-Isomorphismus für Abelsche Gruppen, Math. Z. 179 (1982), 201-212.

16. T. Petrie, Pseudoequivalences of G-manifolds, Proc. Sympos. Pure Math., vol. 32, Amer. Math. Soc., Providence, R.I., 1978, pp. 169-210.

17. G. Segal, Equivariant K-theory, Inst. Hautes Études Sci. Publ. Math. 34 (1968), $129-151$.

18. S. Waner, Equivariant $R O(G)$-graded bordism theories, Topology Appl. 17 (1984), 1-26.

19. A. G. Wassermann, Equivariant differential topology, Topology 8 (1969), 127-150.

20. K. Wirthmüller, Equivariant homology and duality, Manuscripta Math. 11 (1974), 373-390.

\author{
Department of Mathematics, Massachusetts Institute of Technology, \\ CAMBRIDGE, MASSACHUSETTS 02139 \\ Current address: Department of Mathematics, Hofstra University, Hempstead, New York 11374
}

